\title{
A Quasi-Variational Approach for the Dynamic Oligopolistic Market Equilibrium Problem
}

\author{
Annamaria Barbagallo ${ }^{1}$ and Paolo Mauro ${ }^{2}$ \\ ${ }^{1}$ Department of Mathematics and Applications "R. Caccioppoli", University of Naples "Federico II", Via Cintia, 80126 Naples, Italy \\ ${ }^{2}$ Department of Mathematics and Computer Science, University of Catania, Viale A. Doria 6, 95125 Catania, Italy \\ Correspondence should be addressed to Annamaria Barbagallo; annamaria.barbagallo@unina.it
}

Received 18 July 2013; Accepted 19 September 2013

Academic Editor: Abdellah Bnouhachem

Copyright (c) 2013 A. Barbagallo and P. Mauro. This is an open access article distributed under the Creative Commons Attribution License, which permits unrestricted use, distribution, and reproduction in any medium, provided the original work is properly cited.

\begin{abstract}
The paper is concerned with the dynamic oligopolistic market equilibrium problem in the realistic case in which we allow the presence of capacity constraints and production excesses and, moreover, we assume that the production function depends not only on the time but also on the equilibrium distribution. As a consequence, we introduce the generalized dynamic CournotNash principle in the elastic case and prove the equivalence between this equilibrium definition and a suitable evolutionary quasivariational inequality. For completeness we make the analysis of existence, regularity, and sensitivity of the solution. In the end, a numerical example is provided.
\end{abstract}

\section{Introduction}

The aim of the paper is to improve the results obtained in [1] concerning the dynamic oligopolistic market equilibrium problem in presence of production excesses by introducing the dependence on the equilibrium commodity shipment in the production function (see $\mathbb{K}^{*}\left(x^{*}\right)$ in (4)) and, as a consequence, studying the so-called elastic model. This is a more realistic situation since it is reasonable to think that the production function is influenced not only by the time, but also by the evaluation of the amount of commodity shipment, namely, the forecasted equilibrium solution. The presence of production excesses may be well justified in periods of economic crisis, so it is possible that some of the amounts of the commodity available are sold out whereas for a part of the products, an excess of production can occur.

In the last decade a lot of problems considering a feasible set depending on equilibrium solutions have been studied (see, e.g., [2-4]). It is well known that the equilibrium models with fixed constraint sets may be expressed in terms of evolutionary variational inequalities, while models with elastic constraint sets are expressed by evolutionary quasi-variational inequalities. Moreover, the dependence on time leads to considering variational and quasi-variational inequalities in an infinite dimensional setting, for example, a Lebesgue space.

Let us remember that a dynamic oligopolistic market equilibrium problem is the problem of finding a trade equilibrium in a supply-demand market between a finite number of spatially separated firms producing homogeneous goods in a fixed time interval. Moreover, the firms act in a noncooperative behavior. This problem has its origin with Cournot [5]. He considered only two firms and for this reason it was called the duopoly problem. Later, Nash $[6,7]$ extended Cournot's duopoly problem to $n$ agents. A more complete and efficient study was done by Nagurney et al. in [8-11], but the problem was still faced in a static case through a finite dimensional variational approach. Finally, in [12] the time dependence was considered in the model. It allows to explore the change of behavior of equilibrium states for the oligopolistic market models over a finite time interval of interest. As Beckmann and Wallace stressed, for the first time, in [13], "the timedependent formulation of equilibrium problems allows one to explore the dynamics of adjustment processes in which a delay on time response is operating." Of course a delay on time response always happens because the processes do not have an infinite speed. Usually, such adjustment processes can be represented by means of a memory term which depends 
on previous equilibrium solutions according to the Volterra operator (see, e.g., $[14,15])$.

Furthermore, in [16] the authors describe the behavior of the market by using the Lagrange multipliers of the infinite dimensional duality theory developed in [17-21]. Such results make use of the notion of tangent cone, normal cone, and quasi-relative interior of sets (see $[22,23]$ ), important tools to overcome the difficulty of the emptiness of the topological interior of the ordering cone which defines constraints of several infinite dimensional problems (see [24, 25]). Moreover, a sensitivity result has been obtained which states that, under additional assumptions, small changes of the solution happen in correspondence with small changes of the profit function.

Lately, in $[1,26]$, the model presented in [12] has been improved with the addition of production excesses and both production and demand excesses, respectively. Another important question is to find some regularity properties for the solution. In $[1,26]$, the continuity of solution is proved under suitable assumptions, and it results to be very helpful in order to introduce numerical schemes to compute equilibrium solutions (see $[27,28]$ ).

In $[29,30]$, the authors abandon the study of the problem from a producer's point of view whose purpose is to maximize his own profit and focus their attention on the policy-maker's perspective whose aim is to control the commodity exportations by means of the imposition of taxes or incentives and formulate the resulting optimization problem as an inverse variational inequality.

This paper is structured as follows. In Section 2 we present the dynamic oligopolistic market equilibrium problem with the elastic production function and after that we give the definition of equilibrium according to the generalized CournotNash principle. Moreover, we prove the equivalence with a suitable evolutionary quasi-variational inequality. Section 3 is devoted to prove a result of existence of the solution, while in Section 4 Kuratowski's set convergence will be a preliminary property in order to prove the continuity of the equilibrium solution. In Section 5 we establish a sensitivity result that shows how the equilibrium solution can change if the data have been perturbed. In Section 6 a numerical example is provided to make the theoretical model presented in the previous sections more clearer.

\section{Quasi-Variational Inequalities in Dynamic Oligopolistic Markets}

Let us consider $m$ firms $P_{i}, i=1, \ldots, m$, that produce a homogeneous commodity and $n$ demand markets $Q_{j}, j=$ $1, \ldots, n$, that are generally spatially separated. Assume that the homogeneous commodity, produced by the $m$ firms and consumed by the $n$ markets, is involved during a time interval $[0, T], T>0$.

Let $x_{i j}(t), i=1, \ldots, m$ and $j=1, \ldots, n$, denote the nonnegative commodity shipment between the supply market $P_{i}$ and the demand market $Q_{j}$ at the time $t \in[0, T]$. In particular, let us set the vector $x_{i}(t)=\left(x_{i 1}(t), \ldots, x_{i n}(t)\right), i=1, \ldots, m$ and $t \in[0, T]$, as the strategy vector for the firm $P_{i}$.
Let us group the commodity shipments into a matrix function $x:[0, T] \rightarrow \mathbb{R}_{+}^{m n}$ and suppose that $x \in L^{2}([0$, $\left.T], \mathbb{R}_{+}^{m n}\right)$. Furthermore, we assume that the nonnegative commodity shipment $x_{i j}$ between the producer $P_{i}$ and the demand market $Q_{j}$ has to satisfy time-dependent constraints, namely, there exist two nonnegative functions $\underline{x}, \bar{x}:[0, T] \rightarrow \mathbb{R}_{+}^{m n}$ such that

$$
\begin{array}{r}
0 \leq \underline{x}_{i j}(t) \leq x_{i j}(t) \leq \bar{x}_{i j}(t), \quad \forall i=1, \ldots, m, \\
\forall j=1, \ldots, n, \text { a.e. in }[0, T],
\end{array}
$$

and suppose that $\underline{x}, \bar{x} \in L^{2}\left([0, T], \mathbb{R}_{+}^{m n}\right)$.

Let us denote

$$
\begin{aligned}
D=\left\{x \in L^{2}\left([0, T], \mathbb{R}^{m n}\right):\right. & \underline{x}_{i j}(t) \leq x_{i j}(t) \leq \bar{x}_{i j}(t), \\
& \forall i=1, \ldots, m, \\
& \forall j=1, \ldots, n, \text { a.e. in }[0, T]\} .
\end{aligned}
$$

It is easy to verify that $D$ is a nonempty, compact, and convex subset of $L^{2}\left([0, T], \mathbb{R}^{m n}\right)$. Let $p_{i}(t, x(t)), i=1, \ldots, m$, denote the nonnegative commodity output produced by firm $P_{i}$ at the time $t \in[0, T]$. Let us group the production output into a vector function $p:[0, T] \times D \rightarrow \mathbb{R}_{+}^{m}$ and let us suppose that $p \in L^{1}\left([0, T] \times D, \mathbb{R}_{+}^{m}\right)$.

Now, let us introduce the production excesses. Let $\epsilon_{i}(t)$, $i=1, \ldots, m$, be the nonnegative production excess for the commodity of the firm $P_{i}$ at the time $t \in[0, T]$. Let us group the production excess into a vector function $\epsilon:[0, T] \rightarrow \mathbb{R}_{+}^{m}$ and let us assume that $\epsilon \in L^{2}\left([0, T], \mathbb{R}^{m}\right)$.

We consider a formulation of equilibrium problems where the dependence of the production on the unknown solution $x^{*}$ is in the average sense with respect to the time; namely, the following feasibility condition holds:

$$
\begin{array}{r}
\sum_{j=1}^{n} x_{i j}(t)+\epsilon_{i}(t)=\frac{1}{T} \int_{0}^{T} p_{i}\left(t, x^{*}(\tau)\right) d \tau, \\
i=1, \ldots, m, \text { a.e. in }[0, T] .
\end{array}
$$

Hence, condition (3) states that the average of the quantity produced by each firm $P_{i}$, in the time interval $[0, T]$, must be equal to the commodity shipments from that firm to all the demand markets plus the production excess, at the time $t \in[0, T]$. In fact, the production is supposed to depend on the firms' evaluation of the commodity shipments. So one can expect the producers not to evaluate the market practicability instantly, but by an average with respect to the whole time interval. 
The set of feasible vectors $(x, \epsilon) \in L^{2}\left([0, T], \mathbb{R}^{m n+m}\right)$ is then given by the set-valued map $\mathbb{K}: D \rightarrow 2^{L^{2}\left([0, T], \mathbb{R}_{+}^{m n+m}\right)}$ defined as:

$$
\begin{aligned}
& \mathbb{K}^{*}\left(x^{*}\right) \\
& =\left\{(x, \epsilon) \in L^{2}\left([0, T], \mathbb{R}^{m n+m}\right):\right. \\
& \quad \underline{x}_{i j}(t) \leq x_{i j}(t) \leq \bar{x}_{i j}(t), \forall i=1, \ldots, m, \\
& \quad \forall j=1, \ldots, n \text {, a.e. in }[0, T], \\
& \sum_{j=1}^{n} x_{i j}(t)+\epsilon_{i}(t)=\frac{1}{T} \int_{0}^{T} p_{i}\left(t, x^{*}(\tau)\right) d \tau, \\
& \forall i=1, \ldots, m, \text { a.e. in }[0, T], \epsilon_{i}(t) \geq 0, \\
& \forall i=1, \ldots, m \text {, a.e. in }[0, T]\} .
\end{aligned}
$$

Moreover, let us associate with each firm $P_{i}$ a production cost $f_{i}^{*}, i=1, \ldots, m$, and assume that the production cost of a firm $P_{i}$ may depend upon the entire production pattern; namely,

$$
f_{i}^{*}=f_{i}^{*}(t, x(t), \varepsilon(t)) .
$$

Similarly, let us associate with each demand market $Q_{j}$ a demand price for unity of the commodity $d_{j}, j=1, \ldots, n$, and assume that the demand price of a demand market $Q_{j}$ may depend, in general, upon the entire consumption pattern; namely,

$$
d_{j}=d_{j}(t, x(t)) .
$$

Let $g_{i}^{*}, i=1, \ldots, m$, denote the storage cost of the commodity produced by the firm $P_{i}$ and assume that this cost may depend upon the entire production pattern; namely,

$$
g_{i}^{*}=g_{i}^{*}(t, x(t), \varepsilon(t)) .
$$

Finally, let $c_{i j}, i=1, \ldots, m$ and $j=1, \ldots, n$, denote the transaction cost, which includes the transportation cost associated with trading the commodity between firm $P_{i}$ and demand market $Q_{j}$. Here we permit the transaction cost to depend upon the entire shipment pattern; namely,

$$
c_{i j}=c_{i j}(t, x(t)) .
$$

Hence, we have the following mappings:

$$
\begin{aligned}
& f^{*}:[0, T] \times L^{2}\left([0, T], \mathbb{R}_{+}^{m n}\right) \\
& \times L^{2}\left([0, T], \mathbb{R}_{+}^{m}\right) \longrightarrow L^{2}\left([0, T], \mathbb{R}_{+}^{m}\right), \\
& d:[0, T] \times L^{2}\left([0, T], \mathbb{R}_{+}^{m n}\right) \longrightarrow L^{2}\left([0, T], \mathbb{R}_{+}^{n}\right) \text {, } \\
& g^{*}:[0, T] \times L^{2}\left([0, T], \mathbb{R}_{+}^{m n}\right) \\
& \times L^{2}\left([0, T], \mathbb{R}_{+}^{m}\right) \longrightarrow L^{2}\left([0, T], \mathbb{R}_{+}^{m}\right), \\
& c:[0, T] \times L^{2}\left([0, T], \mathbb{R}_{+}^{m n}\right) \longrightarrow L^{2}\left([0, T], \mathbb{R}_{+}^{m n}\right) \text {. }
\end{aligned}
$$

The profit $v_{i}^{*}(t, x(t), \varepsilon(t)), i=1, \ldots, m$, of the firm $P_{i}$ at the time $t \in[0, T]$ is, then,

$$
\begin{aligned}
v_{i}^{*}(t, x(t), \varepsilon(t)) \\
=\sum_{j=1}^{n} d_{j}(t, x(t)) x_{i j}(t) \\
\quad-f_{i}^{*}(t, x(t), \varepsilon(t))-g_{i}^{*}(t, x(t), \varepsilon(t)) \\
\quad-\sum_{j=1}^{n} c_{i j}(t, x(t)) x_{i j}(t) ;
\end{aligned}
$$

namely, it is equal to the price that the demand markets are disposed to pay minus the production costs, the storage costs, and the transportation costs.

By virtue of (3), we can express the nonnegative production excess $\epsilon_{i}(t)$ at the time $t \in[0, T]$ in terms of the integral average of the production function and the commodity shipment. As a consequence, we get

$$
\begin{array}{r}
\sum_{j=1}^{n} x_{i j}(t) \leq \frac{1}{T} \int_{0}^{T} p_{i}\left(t, x^{*}(\tau)\right) d \tau, \\
\forall i=1, \ldots, m, \text { a.e. in }[0, T] .
\end{array}
$$

Then, the production costs and the storage costs, by virtue of (5) and (7), respectively, become

$$
\begin{aligned}
& f_{i}(t, x(t))=f_{i}^{*}(t, x(t), \epsilon(t)), \\
& g_{i}(t, x(t))=g_{i}^{*}(t, x(t), \epsilon(t)),
\end{aligned}
$$

and, analogously, the profit (10) becomes

$$
\begin{aligned}
v_{i}(t, x(t))= & v_{i}^{*}(t, x(t), \epsilon(t)) \\
= & \sum_{j=1}^{n} d_{j}(t, x(t)) x_{i j}(t)-f_{i}(t, x(t)) \\
& -g_{i}(t, x(t))-\sum_{j=1}^{n} c_{i j}(t, x(t)) x_{i j}(t) .
\end{aligned}
$$

As a consequence, the set of feasible vectors $x \in$ $L^{2}\left([0, T], \mathbb{R}^{m n}\right)$ becomes the set-valued map $\mathbb{K}: D \rightarrow$ $2^{L^{2}\left([0, T], \mathbb{R}_{+}^{m n}\right)}$, defined as

$\mathbb{K}\left(x^{*}\right)$

$$
\begin{aligned}
=\left\{x \in L^{2}\left([0, T], \mathbb{R}_{+}^{m n}\right):\right. \\
\\
\quad \underline{x}_{i j}(t) \leq x_{i j}(t) \leq \bar{x}_{i j}(t), \forall i=1, \ldots, m, \\
\quad \forall j=1, \ldots, n, \text { a.e. in }[0, T],
\end{aligned}
$$

$$
\begin{gathered}
\sum_{j=1}^{n} x_{i j}(t) \leq \frac{1}{T} \int_{0}^{T} p_{i}\left(t, x^{*}(\tau)\right) d \tau, \\
\forall i=1, \ldots, m, \text { a.e. in }[0, T]\} .
\end{gathered}
$$


Let us denote $x_{i}=\left\{x_{i j}\right\}_{j=1, \ldots, n}, i=1, \ldots, m$, and $\nabla_{D} v=$ $\left(\partial v_{i} / \partial x_{i j}\right)_{\substack{i=1, \ldots, m \\ j=1, \ldots, n}}$. Let us assume the following assumptions:

(i) $v_{i}(t, x(t))$ is continuously differentiable for each $i=$ $1, \ldots, m$, a.e. in $[0, T]$,

(ii) $\nabla_{D} v=\left(\partial v_{i} / \partial x_{i j}\right)_{\substack{i=1, \ldots, m \\ j=1, \ldots, n}}$ is a Carathéodory function, such that

$\exists \gamma \in L^{2}([0, T]):\left\|\nabla_{D} v(t, x)\right\|_{m n} \leq \gamma(t)+\|x\|_{m n}$, $\forall x \in \mathbb{R}^{m n}$, a.e. in $[0, T]$,

(iii) $v_{i}(t, x(t))$ is pseudoconcave with respect to the variables $x_{i}, i=1, \ldots, m$, a.e. in $[0, T]$.

For the reader's convenience, we recall that a function $v$, continuously differentiable, is called pseudoconcave with respect to $x_{i}, i=1, \ldots, m$, a.e. in $[0, T]$ (see [31]), if the following holds a.e. in $[0, T]$ :

$$
\begin{aligned}
\left\langle\nabla_{D} v_{i}\right. & \left.\left(t, x_{1}, \ldots, x_{i}, \ldots, x_{m}\right), x_{i}-y_{i}\right\rangle \\
& =\sum_{j=1}^{n} \frac{\partial v_{i}(t, x)}{\partial x_{i j}}\left(x_{i j}-y_{i j}\right) \geq 0 \\
& \Longrightarrow v_{i}\left(t, x_{1}, \ldots, x_{i}, \ldots, x_{m}\right) \\
& \geq v_{i}\left(t, x_{1}, \ldots, y_{i}, \ldots, x_{m}\right) .
\end{aligned}
$$

Moreover, we recall that in the Hilbert space $L^{2}([0$, $\left.T], \mathbb{R}^{k}\right)$, we define the canonical bilinear form on $L^{2}([0, T]$, $\left.\mathbb{R}^{k}\right)^{*} \times L^{2}\left([0, T], \mathbb{R}^{k}\right)$ by

$$
\langle\langle\phi, w\rangle\rangle:=\int_{0}^{T}\langle\phi(t), w(t)\rangle d t
$$

where $\phi \in\left(L^{2}\left([0, T], \mathbb{R}^{k}\right)\right)^{*}=L^{2}\left([0, T], \mathbb{R}^{k}\right), w \in L^{2}([0, T]$, $\left.\mathbb{R}^{k}\right)$, and

$$
\langle\phi(t), w(t)\rangle=\sum_{l=1}^{k} \phi_{l}(t) w_{l}(t) .
$$

Now, let us consider the dynamic oligopolistic market, in which the $m$ firms supply the commodity in a noncooperative fashion, each one trying to maximize its own profit at the time $t \in[0, T]$. We seek to determine a nonnegative commodity distribution matrix function $x^{*}$ for which the $m$ firms and the $n$ demand markets will be in a state of equilibrium according to the dynamic Cournot-Nash principle.

Definition 1. $x^{*} \in \mathbb{K}\left(x^{*}\right)$ is a dynamic oligopolistic market equilibrium in presence of production excesses if and only if for each $i=1, \ldots, m$ and a.e. in $[0, T]$

$$
v_{i}\left(t, x^{*}(t)\right) \geq v_{i}\left(t, x_{i}(t), \widehat{x}_{i}^{*}(t)\right), \quad \text { a.e. in }[0, T],
$$

where $x_{i}(t)=\left(x_{i 1}(t), \ldots, x_{i n}(t)\right)$ and $\widehat{x}_{i}^{*}(t)=\left(x_{1}^{*}(t), \ldots\right.$, $\left.x_{i-1}^{*}(t), x_{i+1}^{*}(t), \ldots, x_{m}^{*}(t)\right)$, for $i=1, \ldots, m$, a.e. in $[0, T]$.

Definition 1 states that each firm $P_{i}$ maximizes its own profit, at the time $t \in[0, T]$, considering the given optimal strategy $\widehat{x}_{i}^{*}(t)$ of the other firms.
Theorem 2. Suppose that assumptions (i), (ii), and (iii) are satisfied. Then, $x^{*} \in \mathbb{K}\left(x^{*}\right)$ is a dynamic oligopolistic market equilibrium according to Definition 1 if and only if it satisfies the evolutionary quasi-variational inequality

$$
\int_{0}^{T} \sum_{i=1}^{m} \sum_{j=1}^{n}\left(-\frac{\partial v_{i}\left(t, x^{*}(t)\right)}{\partial x_{i j}}\right)\left(x_{i j}(t)-x_{i j}^{*}(t)\right) d t \geq 0
$$

$$
\forall x \in \mathbb{K}\left(x^{*}\right)
$$

Proof. First of all, let us prove that the evolutionary quasivariational inequality (20), that we can write as follows:

$$
\begin{aligned}
& \int_{0}^{T} \sum_{i=1}^{m} \sum_{j=1}^{n}-\frac{\partial v_{i}\left(t, x^{*}(t)\right)}{\partial x_{i j}}\left(x_{i j}(t)-x_{i j}^{*}(t)\right) d t \\
&=\left\langle\left\langle-\nabla_{D} v\left(x^{*}\right), x-x^{*}\right\rangle\right\rangle \\
&=\int_{0}^{T}\left\langle-\nabla_{D} v\left(t, x^{*}(t)\right), x(t)-x^{*}(t)\right\rangle d t \geq 0 \\
& \forall x \in \mathbb{K}\left(x^{*}\right),
\end{aligned}
$$

is equivalent to the following point-to-point quasi-variational inequality:

$$
\begin{array}{r}
\left\langle-\nabla_{D} v\left(t, x^{*}(t)\right), x(t)-x^{*}(t)\right\rangle \\
=\sum_{i=1}^{m} \sum_{j=1}^{n}-\frac{\partial v_{i}\left(t, x^{*}(t)\right)}{\partial x_{i j}}\left(x_{i j}(t)-x_{i j}^{*}(t)\right) \geq 0 \\
\forall x(t) \in \mathbb{K}\left(t, x^{*}\right), \text { a.e. in }[0, T],
\end{array}
$$

where

$$
\begin{aligned}
& \mathbb{K}\left(t, x^{*}\right) \\
& =\left\{x(t) \in \mathbb{R}_{+}^{m n}: \underline{x}_{i j}(t) \leq x_{i j}(t) \leq \bar{x}_{i j}(t),\right. \\
& \forall i=1, \ldots, m, \forall j=1, \ldots, n, \\
& \sum_{j=1}^{n} x_{i j}(t) \leq \frac{1}{T} \int_{0}^{T} p_{i}\left(t, x^{*}(\tau)\right) d \tau, \\
& \forall i=1, \ldots, m\} .
\end{aligned}
$$

In fact, let us suppose by absurdum that (22) does not hold, namely, $\exists \bar{x}(t) \in \mathbb{K}\left(x^{*}\right), \exists I \subseteq[0, T]$ with $m(I)>0$ such that

$$
\left\langle-\nabla_{D^{v}}\left(t, x^{*}(t)\right), \bar{x}(t)-x^{*}(t)\right\rangle<0 \quad \text { a.e. in } I \text {. }
$$

Let us choose, now,

$$
x(t)= \begin{cases}x^{*}(t), & \text { in }[0, T] \backslash I, \\ \bar{x}(t), & \text { in } I .\end{cases}
$$


Hence, let us consider

$$
\begin{aligned}
& \left\langle\left\langle-\nabla_{D^{2}} v\left(t, x^{*}(t)\right), x-x^{*}\right\rangle\right\rangle \\
& =\int_{[0, T] \backslash I}\left\langle-\nabla_{D} v\left(t, x^{*}(t)\right), x(t)-x^{*}(t)\right\rangle d t \\
& \quad+\int_{I}\left\langle-\nabla_{D} v\left(t, x^{*}(t)\right), \bar{x}(t)-x^{*}(t)\right\rangle d t<0,
\end{aligned}
$$

that is a contradiction. The vice versa is immediate.

So the equivalence between the evolutionary quasivariational inequalities (20) and (22) is proved.

Let us prove, now, the equivalence between the dynamic Cournot-Nash principle and the evolutionary quasi-variational inequality (20).

Let us suppose that $x^{*} \in \mathbb{K}\left(x^{*}\right)$ is an equilibrium point according to Definition 1; namely,

$$
\begin{array}{r}
v_{i}\left(t, x^{*}(t)\right) \geq v_{i}\left(t, x(t), \hat{x}^{*}(t)\right) \quad \forall x(t) \in \mathbb{K}\left(t, x^{*}\right), \\
\text { a.e. in }[0, T], \forall i=1, \ldots, m .
\end{array}
$$

For well known theorems of optimization, we have that the necessary and sufficient condition to get (27) is that for all $i=1, \ldots, m$, for all $x(t) \in \mathbb{K}\left(t, x^{*}\right)$, a.e. in $[0, T]$

$$
\begin{aligned}
\left\langle-\nabla_{D} v_{i}\left(t, x^{*}(t)\right), x_{i}(t)-x_{i}^{*}(t)\right\rangle \\
=\sum_{j=1}^{n}-\frac{\partial v_{i}\left(t, x^{*}(t)\right)}{\partial x_{i j}}\left(x_{i j}(t)-x_{i j}^{*}(t)\right) \geq 0 .
\end{aligned}
$$

By assumption that $\nabla_{D} v_{i}$ is a Carathéodory function such that

$$
\begin{aligned}
\exists \gamma \in L^{2}([0, T]): & \left\|\nabla_{D} v_{i}(t, x)\right\|_{m n} \\
& \leq \gamma(t)+\|x\|_{m n}, \quad \forall x \in \mathbb{R}^{m n}, \text { a.e. in }[0, T],
\end{aligned}
$$

moreover, $x$ and $x^{*} \in L^{2}\left([0, T], \mathbb{R}^{m n}\right)$, so we have

$$
t \longrightarrow\left\langle-\nabla_{D} v_{i}\left(t, x^{*}(t)\right), x_{i}(t)-x_{i}^{*}(t)\right\rangle \in L^{2}([0, T], \mathbb{R}) .
$$

Then, we get

$$
\left\langle\left\langle-\nabla_{D} v_{i}\left(t, x^{*}(t)\right), x_{i}-x_{i}^{*}\right\rangle\right\rangle \geq 0 \quad \forall x \in \mathbb{K}\left(x^{*}\right),
$$

from which, by summing up each firm $P_{i}$, for $i=1, \ldots, m$, we obtain

$$
\begin{aligned}
\sum_{i=1}^{m} & \left\langle\left\langle-\nabla_{D} v_{i}\left(t, x^{*}(t)\right), x-x^{*}\right\rangle\right\rangle \\
& =\left\langle\left\langle-\nabla_{D} v\left(t, x^{*}(t)\right), x_{i}-x_{i}^{*}\right\rangle\right\rangle \geq 0 \quad \forall x \in \mathbb{K}\left(x^{*}\right) .
\end{aligned}
$$

Vice versa, let us suppose that $x^{*}(t)$ is a solution to evolutionary quasi-variational inequality (20), but not an equilibrium solution according to the dynamic CournotNash principle, namely, $\exists I \subseteq[0, T]$ with $m(I)>0, \exists \bar{i} \in$ $\{1, \ldots, m\}$ and $\exists \tilde{x}_{\bar{i}}$ such that

$$
v_{\bar{i}}\left(t, x^{*}(t)\right)<v_{\bar{i}}\left(t, \tilde{x}_{i}(t), \widehat{x}^{*}(t)\right) \quad \text { in } I .
$$

Since the profit function $v_{\bar{i}}(t, x(t))$ is pseudoconcave with respect to $x_{\bar{i}}$, we get

$$
\left\langle-\nabla_{D} v_{\bar{i}}\left(t, x^{*}(t)\right), x_{\bar{i}}^{*}(t)-\tilde{x}_{\bar{i}}(t)\right\rangle<0 \quad \text { in } I .
$$

If we choose $x \in \mathbb{K}\left(x^{*}\right)$ such that

$$
x_{i}(t)= \begin{cases}x_{i}^{*}(t) \text { in }[0, T] \backslash I, & \forall i=1, \ldots, m, \\ x_{i}^{*}(t) \text { in } I, & \text { if } i \neq \bar{i}, \\ \tilde{x}_{i} \text { in } I, & \text { if } i=\bar{i},\end{cases}
$$

then

$$
\begin{aligned}
\int_{0}^{T} & \left\langle-\nabla_{D} v\left(t, x^{*}(t)\right), x(t)-x^{*}(t)\right\rangle d t \\
& =\int_{I}\left\langle-\nabla_{D} v_{\bar{i}}\left(t, x^{*}(t)\right), \widetilde{x}_{\bar{i}}(t)-x_{\bar{i}}^{*}(t)\right\rangle d t<0,
\end{aligned}
$$

so we get the contradiction.

\section{An Existence Theorem for Equilibrium Solutions}

Now, we prove an existence result for the equilibrium solution to the dynamic elastic oligopolistic market equilibrium problem. To this aim, we recall a general existence result for solutions to quasi-variational inequalities in topological linear locally convex Hausdorff spaces due to Tan [32].

Theorem 3. Let $X$ be a topological linear locally convex Hausdorff space and let $D$ be a convex compact nonempty subset of $X$. Let $C: D \rightarrow 2^{X^{*}}$ be an upper semicontinuous multivalued mapping with $C(x), x \in D$, convex compact nonempty, let $\mathbb{K}: D \rightarrow 2^{D}$ be a closed lower semicontinuous multivalued mapping with $\mathbb{K}(x), x \in D$, convex compact nonempty, and let $\varphi: D \rightarrow \mathbb{R}$ be a proper convex lower semicontinuous function. Then, there exists $x^{*} \in D$ such that:

(i) $x^{*} \in \mathbb{K}\left(x^{*}\right)$,

(ii) there exists $y^{*} \in \mathbb{K}\left(x^{*}\right)$ for which

$$
\left\langle x-x^{*}, y^{*}\right\rangle+\varphi(x)-\varphi\left(x^{*}\right) \geq 0, \quad \forall x \in \mathbb{K}\left(x^{*}\right) .
$$

Now, we are able to prove our main result.

Theorem 4. Let $v:[0, T] \rightarrow \mathbb{R}^{m}$ and $p:[0, T] \rightarrow \mathbb{R}^{m}$ be two vector functions such that assumptions (i) and (iii) are satisfied and

(I) $\nabla_{D} v(t, x)$ is measurable in $t$, for all $x \in \mathbb{R}_{+}^{m n}$, continuous in $x$, a.e. in $[0, T]$, such that $\exists \gamma \in L^{2}([0, T])$ : $\left\|\nabla_{D} v(t, x)\right\| \leq \gamma(t)+\|x\|$, for all $x \in \mathbb{R}_{+}^{m n}$, a.e. in $[0, T]$; 
(II) $p(t, x)$ is measurable in $t$, for all $x \in \mathbb{R}_{+}^{m n}$, continuous in $x$, a.e. in $[0, T]$, such that $\exists \phi \in L^{1}([0, T])$ : $\|p(t, x)\| \leq \phi(t)+\|x\|^{2}$, for all $x \in \mathbb{R}_{+}^{m n}$, a.e. in $[0, T] ;$

(III) $\exists v(t) \geq 0$, a.e. in $[0, T], \eta \in L^{\infty}([0, T])$ such that

$$
\begin{array}{r}
\left\|p\left(t, x_{1}\right)-p\left(t, x_{2}\right)\right\| \leq \eta(t)\left\|x_{1}-x_{2}\right\|, \\
\forall x_{1}, x_{2} \in \mathbb{R}_{+}^{m n} \text {, a.e. in }[0, T] .
\end{array}
$$

Then, evolutionary quasi-variational inequality (20) admits a solution.

Proof. At first, observe that under the hypotheses (I) and (II) and if $x \in L^{2}\left([0, T], \mathbb{R}_{+}^{m n}\right)$,

$$
\begin{gathered}
t \longmapsto \nabla_{D} v(t, x(t)) \in L^{2}\left([0, T], \mathbb{R}_{+}^{m n}\right) \\
t \longmapsto p(t, x(t)) \in L^{1}\left([0, T], \mathbb{R}_{+}^{m}\right)
\end{gathered}
$$

Moreover, by (I) and (II) it follows that $\nabla_{D} v$ and $p$ belong to the class of nemytskii operators. Therefore if $\left\{x^{k}\right\}_{k \in \mathbb{N}}$ is a sequence such that $x^{k} \rightarrow x$, in $L^{2}\left([0, T], \mathbb{R}^{m n}\right)$, we have

$$
\begin{gathered}
\left\|\nabla_{D} v\left(t, x^{k}\right)-\nabla_{D^{v}} v(t, x)\right\|_{L^{2}} \longrightarrow 0, \\
\left\|p\left(t, x^{k}\right)-p(t, x)\right\|_{L^{1}} \longrightarrow 0,
\end{gathered}
$$

where the functions $\nabla_{D} v$ and $p$ are $L^{2}$ - and $L^{1}$-continuous, respectively.

Now, in order to show that $\mathbb{K}\left(x^{*}\right)$ is a closed multifunction, we prove that the following condition holds. For every two arbitrary sequences $\left\{x^{k}\right\}_{k \in \mathbb{N}}$ and $\left\{y^{k}\right\}_{k \in \mathbb{N}}$ such that $x^{k} \rightarrow$ $x$ and $y^{k} \rightarrow y$ in $L^{2}\left([0, T], \mathbb{R}^{m n}\right)$, with $y^{k} \in \mathbb{K}\left(x^{k}\right), \forall n \in \mathbb{N}$, then $y \in \mathbb{K}(x)$. To this aim, let us consider two arbitrary convergent sequences in $L^{2}\left([0, T], \mathbb{R}^{m n}\right),\left\{x^{k}\right\}_{k \in \mathbb{N}}$ and $\left\{y^{k}\right\}_{k \in \mathbb{N}}$ to $x$ and $y$, respectively. Since $y^{k} \in \mathbb{K}\left(x^{k}\right), \underline{x}_{i j}(t) \leq y_{i j}^{k}(t) \leq$ $\bar{x}_{i j}(t)$, for $i=1, \ldots, m, j=1, \ldots, n$, and a.e. in $[0, T]$, and the convergence of the sequence $\left\{y^{k}\right\}_{k \in \mathbb{N}}$ in $L^{2}\left([0, T], \mathbb{R}^{m n}\right)$ implies that also $y$ satisfies the capacity constraints.

Moreover, the following relationship holds:

$$
\begin{array}{r}
\sum_{j=1}^{m} y_{i j}^{k}(t) \leq \frac{1}{T} \int_{0}^{T} p_{i}\left(t, x^{k}(\tau)\right) d \tau \\
i=1, \ldots, m, \text { a.e. in }[0, T] .
\end{array}
$$

The left-hand side converges almost everywhere to $\sum_{j=1}^{m} y_{i j}(t)$; for the right-hand side, meanwhile, $i=1, \ldots, m$, we have

$$
\begin{gathered}
\sup _{[0, T]}\left\|\int_{0}^{T} p\left(t, x^{k}(\tau)\right) d \tau-\int_{0}^{T} p(t, x(\tau)) d \tau\right\| \\
\quad \leq \sup _{[0, T]} \int_{0}^{T}\left\|p\left(t, x^{k}(\tau)\right)-p(t, x(\tau))\right\| d \tau \\
\quad \leq\|\eta\|_{L^{\infty}([0, T])} \int_{0}^{T}\left\|x^{k}(\tau)-x(\tau)\right\| d \tau .
\end{gathered}
$$

By considering that the convergence of $\left\{x^{k}\right\}$ in $L^{2}$ implies the convergence even in $L^{1}$, hence the sequence $\left\{(1 / T) \int_{0}^{T} p_{i}(t\right.$, $\left.\left.x^{k}(\tau)\right) d \tau\right\}_{k \in \mathbb{N}}$ converges uniformly to $(1 / T) \int_{0}^{T} p_{i}(t, x(\tau)) d \tau$ in $L^{1}\left([0, T], \mathbb{R}^{m}\right)$.

Now, let us show the lower semicontinuity of the multifunction $\mathbb{K}$. To this aim it suffices to prove that for every $\left\{x^{k}\right\}_{k \in \mathbb{N}}$ such that $x^{k} \rightarrow x$, in $L^{2}\left([0, T], \mathbb{R}^{m n}\right)$, and for every $y \in \mathbb{K}(x)$, there exists a sequence $\left\{y^{k}\right\}_{k \in \mathbb{N}}$ such that $y^{k} \rightarrow y$, in $L^{2}\left([0, T], \mathbb{R}^{m n}\right)$, with $y^{k} \in \mathbb{K}\left(x^{k}\right)$, for all $k \in \mathbb{N}$.

Let $\left\{x^{k}\right\}_{k \in \mathbb{N}}$ be an arbitrary sequence such that $x^{k} \rightarrow x$, in $L^{2}\left([0, T], \mathbb{R}^{m n}\right)$, and let $y \in \mathbb{K}(x)$. Let us note that, for $i=$ $1, \ldots, m$ and $j=1, \ldots, n$, and if

$$
\begin{aligned}
a_{i j}^{k}(t)= & y_{i j}(t)-\underline{x}_{i j}(t)+\frac{1}{n T} \\
& \times\left[\int_{0}^{T} p_{i}(t, x(\tau)) d \tau-\int_{0}^{T} p_{i}\left(t, x^{k}(\tau)\right) d \tau\right],
\end{aligned}
$$

we obtain, by virtue of the uniform convergence of $\left\{(1 / T) \int_{0}^{T} p_{i}\left(t, x^{k}(\tau)\right) d \tau\right\}_{k \in \mathbb{N}}$ to $(1 / T) \int_{0}^{T} p_{i}(t, x(\tau)) d \tau \quad$ in $L^{1}\left([0, T], \mathbb{R}^{m}\right)$, that

$$
\lim _{k \rightarrow+\infty} a_{i j}^{k}(t)=y_{i j}(t)-\underline{x}_{i j}(t) \geq 0, \quad \text { a.e. in }[0, T] .
$$

As a consequence, there exists an index $v$ such that for $k>v$ one has, for all $i=1, \ldots, m$, for all $j=1, \ldots, n$,

$$
a_{i j}^{k}(t) \geq 0, \quad \text { a.e. in }[0, T] \text {. }
$$

Then, we consider the sequence $\left\{y^{k}\right\}_{k \in \mathbb{N}}$ such that

(i) for $k>v$, for all $i=1, \ldots, m$, for all $j=1, \ldots, n$,

$$
\begin{array}{r}
y_{i j}^{k}(t)=\underline{x}_{i j}(t)+\min \left\{\bar{x}_{i j}(t)-\underline{x}_{i j}(t), a_{i j}^{k}(t)\right\}, \\
\text { a.e. in }[0, T],
\end{array}
$$

(ii) for $k \leq v$, for all $i=1, \ldots, m$, for all $j=1, \ldots, n$,

$$
y_{i j}^{k}(t)=P_{\mathbb{K}\left(x^{k}\right)} y_{i j}(t), \quad \text { a.e. in }[0, T],
$$

where $P_{\mathbb{K}\left(x^{k}\right)}(\cdot)$ denotes the Hilbertian projection on $\mathbb{K}\left(x^{k}\right)$.

It is easy to verify that if $k \leq v$, for (47), $y^{k} \in \mathbb{K}\left(x^{k}\right)$. Instead, for $k>v$, since for $(45), \min \left\{\bar{x}_{i j}(t)-\underline{x}_{i j}(t), a_{i j}^{k}(t)\right\} \geq 0$, for all $i=1, \ldots, m$, for all $j=1, \ldots, n$, a.e. in $[0, T]$,

$y_{i j}^{k}(t) \geq \underline{x}_{i j}(t), \quad \forall i=1, \ldots, m, \forall j=1, \ldots, n$, a.e. in $[0, T]$.

Moreover, since $\min \left\{\bar{x}_{i j}(t)-\underline{x}_{i j}(t), a_{i j}^{k}(t)\right\} \leq \bar{x}_{i j}(t)-\underline{x}_{i j}(t)$, for all $i=1, \ldots, m$, for all $j=1, \ldots, n$, a.e. in $[0, T]$, we have

$$
y_{i j}^{k}(t) \leq \bar{x}_{i j}(t), \quad \forall i=1, \ldots, m, \forall j=1, \ldots, n \text {, a.e. in }[0, T] .
$$


Finally, we get

$$
\begin{aligned}
& \sum_{j=1}^{n} y_{i j}^{k}(t) \leq \sum_{j=1}^{n} y_{i j}(t)+\frac{1}{T} \int_{0}^{T} p_{i}\left(t, x^{k}(\tau)\right) d \tau \\
& \quad-\frac{1}{T} \int_{0}^{T} p_{i}(t, x(\tau)) d \tau \\
& \leq \frac{1}{T} \int_{0}^{T} p_{i}(t, x(\tau)) d \tau+\frac{1}{T} \int_{0}^{T} p_{i}\left(t, x^{k}(\tau)\right) d \tau \\
& \quad-\frac{1}{T} \int_{0}^{T} p_{i}(t, x(\tau)) d \tau \\
& =\frac{1}{T} \int_{0}^{T} p_{i}\left(t, x^{k}(\tau)\right) d \tau, \quad \forall i=1, \ldots, m \text {, a.e. in }[0, T]
\end{aligned}
$$

Hence, we can conclude that $y^{k}$ belongs to $\mathbb{K}\left(x^{k}\right)$, for all $k \in$ $\mathbb{N}$.

Let us prove now the convergence of $\left\{y^{k}\right\}_{k \in \mathbb{N}}$ to $y$ in $L^{2}\left([0, T], \mathbb{R}^{m n}\right)$. Let us observe that

$$
\begin{aligned}
\lim _{k \rightarrow+\infty} \min & \left\{\bar{x}_{i j}(t)-\underline{x}_{i j}(t), a_{i j}^{k}(t)\right\} \\
& =y_{i j}(t)-\underline{x}_{i j}(t), \quad \text { a.e. in }[0, T] .
\end{aligned}
$$

As a consequence, we have that the sequence $\left\{y^{k}\right\}_{k \in \mathbb{N}}$ converges to $y$. It is easy to show that $\mathbb{K}(x)$ is a closed, bounded, and convex subset of $D$ and since the space $D$ is compact, $\mathbb{K}(x)$, for all $x \in D$, is compact too. As a consequence, all the hypotheses of Theorem 3 are satisfied and the existence of at least one solution is guaranteed.

\section{Regularity Results for Equilibrium Solutions}

In this section, we study the assumptions under which the continuity of solutions to evolutionary quasi-variational inequality, which expresses the equilibrium condition for the dynamic elastic oligopolistic market equilibrium problem in presence of production excesses, is ensured.

4.1. Set Convergence. First of all, we recall the notion of Kuratowski's set convergence that has an important role in order to establish regularity results. The classical notion of convergence for subsets of a given metric space $(X, d)$ is introduced in the 1950s by Kuratowski (see [33]; see also $[34,35])$.

Let $\left\{\mathbb{K}_{n}\right\}_{n \in \mathbb{N}}$ be a sequence of subsets of $X$. Recall that

$$
\begin{aligned}
& d-\frac{\lim }{n} \mathbb{K}_{n}=\left\{x \in X: \exists\left\{x_{n}\right\}_{n \in \mathbb{N}}\right. \\
& \left.\quad \text { eventually in } \mathbb{K}_{n} \text { such that } x_{n} \stackrel{d}{\longrightarrow} x\right\}, \\
& d-\varlimsup_{n} \mathbb{K}_{n}=\left\{x \in X: \exists\left\{x_{n}\right\}_{n \in \mathbb{N}}\right.
\end{aligned}
$$$$
\text { frequently in } \left.\mathbb{K}_{n} \text { such that } x_{n} \stackrel{d}{\longrightarrow} x\right\} \text {, }
$$

where eventually means that there exists $\delta \in \mathbb{N}$ such that $x_{n} \in \mathbb{K}_{n}$ for any $n \geq \delta$ and frequently means that there exists an infinite subset $N \subseteq \mathbb{N}$ such that $x_{n} \in \mathbb{K}_{n}$ for any $n \in N$ (in this last case, according to the notation given above, we also write that there exists a subsequence $\left\{x_{k_{n}}\right\}_{n \in \mathbb{N}} \subseteq\left\{x_{n}\right\}_{n \in \mathbb{N}}$ such that $\left.x_{k_{n}} \in \mathbb{K}_{k_{n}}\right)$.

In the following, we recall Kuratowski's set convergence.

Definition 5. We say that $\left\{\mathbb{K}_{n}\right\}$ converges to some subset $\mathbb{K} \subseteq$ $X$ in Kuratowski's sense and we briefly write $\mathbb{K}_{n} \rightarrow \mathbb{K}$, if $d-$ $\underline{\lim }_{n} \mathbb{K}_{n}=d-\overline{\lim }_{n} \mathbb{K}_{n}=\mathbb{K}$. Thus, in order to verify that $\mathbb{K}_{n} \rightarrow$ $\mathbb{K}$, it suffices to check that

(i) $d-\varlimsup_{n} \mathbb{K}_{n} \subseteq \mathbb{K}$, that is, for any sequence $\left\{x_{n}\right\}_{n \in \mathbb{N}}$ frequently in $\mathbb{K}_{n}$ such that $x_{n} \stackrel{d}{\rightarrow} x$ for some $x \in S$, then $x \in \mathbb{K}$;

(ii) $\mathbb{K} \subset d-\underline{\lim }_{n} \mathbb{K}_{n}$, that is, for any $x \in \mathbb{K}$ there exists a sequence $\left\{x_{n}\right\}_{n \in \mathbb{N}}$ eventually in $\mathbb{K}_{n}$ such that $x_{n} \stackrel{d}{\rightarrow} x$.

The below lemma establishes that the feasible set $\mathbb{K}$ of the dynamic elastic oligopolistic market equilibrium problem in the presence of production excesses satisfies the property of Kuratowski's set convergence.

Lemma 6. Let $\underline{x}, \bar{x} \in C^{0}\left([0, T], \mathbb{R}_{+}^{m n}\right)$, let $p \in C^{0}([0, T] \times$ $\mathbb{R}_{+}^{m n}, \mathbb{R}_{+}^{m}$ ) be such that

$$
\begin{array}{r}
\exists \phi \in C^{0}\left([0, T], \mathbb{R}_{+}\right):\|p(t, x)\| \leq \phi(t)+\|x\|^{2}, \\
\forall x \in \mathbb{R}_{+}^{m n} \text {, in }[0, T],
\end{array}
$$

and let $\left\{t_{k}\right\}_{k \in \mathbb{N}}$ be a sequence such that $t_{k} \rightarrow t$, with $t \in[0, T]$, as $k \rightarrow+\infty$. Then, the sequence of sets

$$
\begin{gathered}
\mathbb{K}\left(t_{k}, x^{*}\right)=\left\{x\left(t_{k}\right) \in \mathbb{R}^{m n}: \underline{x}_{i j}\left(t_{k}\right) \leq x_{i j}\left(t_{k}\right) \leq \bar{x}_{i j}\left(t_{k}\right),\right. \\
\forall i=1, \ldots, m, \forall j=1, \ldots, n, \\
\sum_{j=1}^{n} x_{i j}\left(t_{k}\right) \leq \frac{1}{T} \int_{0}^{T} p_{i}\left(t_{k}, x^{*}(\tau)\right) d \tau, \\
\forall i=1, \ldots, m\},
\end{gathered}
$$

for all $k \in \mathbb{N}$, converges to

$$
\begin{gathered}
\mathbb{K}\left(t, x^{*}\right)=\left\{x(t) \in \mathbb{R}^{m n}: \underline{x}_{i j}(t) \leq x_{i j}(t) \leq \bar{x}_{i j}(t),\right. \\
\forall i=1, \ldots, m, \forall j=1, \ldots, n, \\
\sum_{j=1}^{n} x_{i j}(t) \leq \frac{1}{T} \int_{0}^{T} p_{i}\left(t, x^{*}(\tau)\right) d \tau, \\
\forall i=1, \ldots, m\},
\end{gathered}
$$


Proof. Firstly, we prove condition (K1). Let $\left\{t_{k}\right\}_{k \in \mathbb{N}}$ be a sequence such that $t_{k} \rightarrow t$, with $t \in[0, T]$ as $k \rightarrow+\infty$. Making use of the continuity assumptions on $x, \bar{x}$, and $p$, we get $\underline{x}\left(t_{k}\right) \rightarrow \underline{x}(t), \bar{x}\left(t_{k}\right) \rightarrow \bar{x}(t)$, and $p\left(t_{k}, y\right) \rightarrow p(t, y)$ as $k \rightarrow+\infty$, respectively. Furthermore,

$$
\exists \phi \in C^{0}\left([0, T], \mathbb{R}_{+}\right):\left\|p\left(t, x^{*}(\tau)\right)\right\| \leq \phi(t)+\left\|x^{*}(\tau)\right\|^{2},
$$

for $t \in[0, T]$ and $\tau \in[0, T]$. Since $\phi \in C^{0}([0, T])$ and $x^{*} \in$ $L^{2}\left([0, T], \mathbb{R}_{+}^{m n}\right)$, then we have for $t \in[0, T]$ and $\tau \in[0, T]$

$$
\left\|p\left(t, x^{*}(\tau)\right)\right\| \leq \phi(t)+\left\|x^{*}(\tau)\right\|^{2} \in L^{1}([0, T]),
$$

and by virtue of the continuity of $p$ with respect to the first variable we also obtain

$$
\lim _{n \rightarrow+\infty} p\left(t_{n}, x^{*}(\tau)\right)=p\left(t, x^{*}(\tau)\right),
$$

for $\tau \in[0, T]$ and $x^{*} \in L^{2}\left([0, T], \mathbb{R}_{+}^{m n}\right)$. Taking into account a well known generalization of Lebesgue's theorem,

$$
\lim _{n \rightarrow+\infty} \int_{0}^{T} p\left(t_{n}, x^{*}(\tau)\right) d \tau=\int_{0}^{T} p\left(t, x^{*}(\tau)\right) d \tau
$$

for every $x^{*} \in L^{2}\left([0, T], \mathbb{R}_{+}^{m n}\right)$.

Let $x(t) \in \mathbb{K}\left(t, x^{*}\right)$ be fixed and let us note that, for $i=$ $1, \ldots, m$ and $j=1, \ldots, n$, and if

$$
\begin{aligned}
a_{i j}\left(t_{k}\right)= & x_{i j}(t)-\underline{x}_{i j}\left(t_{k}\right)+\frac{1}{n T} \\
& \times\left[\int_{0}^{T} p_{i}\left(t_{k}, x^{*}(\tau)\right) d \tau-\int_{0}^{T} p_{i}\left(t, x^{*}(\tau)\right) d \tau\right],
\end{aligned}
$$

we have

$$
\lim _{k \rightarrow+\infty} a_{i j}\left(t_{k}\right)=x_{i j}(t)-\underline{x}_{i j}(t) \geq 0 .
$$

As a consequence, there exists an index $v$ such that for $k>v$, for all $i=1, \ldots, m$, for all $j=1, \ldots, n$,

$$
a_{i j}\left(t_{k}\right) \geq 0 \text {. }
$$

As a consequence, we consider the sequence $\left\{x\left(t_{k}\right)\right\}_{k \in \mathbb{N}}$ such that

(i) for $k>v$, for all $i=1, \ldots, m$, for all $j=1, \ldots, n$,

$$
\begin{aligned}
x_{i j}\left(t_{k}\right)=\underline{x}_{i j}\left(t_{k}\right)+\min \left\{x_{i j}(t)-\underline{x}_{i j}(t),\right. \\
\left.\bar{x}_{i j}\left(t_{k}\right)-\underline{x}_{i j}\left(t_{k}\right), a_{i j}\left(t_{k}\right)\right\},
\end{aligned}
$$

(ii) for $k \leq v$, for all $i=1, \ldots, m$, for all $j=1, \ldots, n$,

$$
x_{i j}\left(t_{k}\right)=P_{\mathbb{K}\left(t_{k}, x^{*}\right)} x_{i j}(t),
$$

where $P_{\mathbb{K}\left(t_{k}, x^{*}\right)}(\cdot)$ denotes the Hilbertian projection on $\mathbb{K}\left(t_{k}, x^{*}\right)$.

Obviously if $k \leq v$, for (64) we have $x\left(t_{k}\right) \in \mathbb{K}\left(t_{k}, x^{*}\right)$. Instead, for $k>v$, since for $(62), \min \left\{x_{i j}(t)-\underline{x}_{i j}(t), \bar{x}_{i j}\left(t_{k}\right)-\right.$ $\left.\underline{x}_{i j}\left(t_{k}\right), a_{i j}\left(t_{k}\right)\right\} \geq 0$, for all $i=1, \ldots, m$, for all $j=1, \ldots, n$, we get

$$
\underline{x}_{i j}\left(t_{k}\right) \leq x_{i j}\left(t_{k}\right), \quad \forall i=1, \ldots, m, \forall j=1, \ldots, n .
$$

Moreover, since $\min \left\{x_{i j}(t)-\underline{x}_{i j}(t), \bar{x}_{i j}\left(t_{k}\right)-\underline{x}_{i j}\left(t_{k}\right), a_{i j}\left(t_{k}\right)\right\} \leq$ $\bar{x}_{i j}\left(t_{k}\right)-\underline{x}_{i j}\left(t_{k}\right)$, for all $i=1, \ldots, m$, for all $j=1, \ldots, n$, we have

$$
x_{i j}\left(t_{k}\right) \leq \bar{x}_{i j}\left(t_{k}\right), \quad \forall i=1, \ldots, m, \forall j=1, \ldots, n .
$$

Since

$$
\begin{aligned}
\min \left\{x_{i j}(t)-\underline{x}_{i j}(t), \bar{x}_{i j}\left(t_{k}\right)-\underline{x}_{i j}\left(t_{k}\right), a_{i j}\left(t_{k}\right)\right\} \\
\leq a_{i j}\left(t_{k}\right)=x_{i j}(t)-\underline{x}_{i j}\left(t_{k}\right) \\
+\left[\frac{1}{n T} \int_{0}^{T} p_{i}\left(t_{k}, x^{*}(\tau)\right) d \tau-\int_{0}^{T} p_{i}\left(t, x^{*}(\tau)\right) d \tau\right] \\
\forall i=1, \ldots, m, \forall j=1, \ldots, n,
\end{aligned}
$$

we have

$$
\begin{aligned}
& x_{i j}\left(t_{k}\right) \leq x_{i j}(t)+\frac{1}{n T} \\
& \times\left[\int_{0}^{T} p_{i}\left(t_{k}, x^{*}(\tau)\right) d \tau-\int_{0}^{T} p_{i}\left(t, x^{*}(\tau)\right) d \tau\right], \\
& \forall i=1, \ldots, m, \forall j=1, \ldots, n .
\end{aligned}
$$

Then, taking into account (68), we obtain

$$
\begin{aligned}
\sum_{j=1}^{n} x_{i j}( & \left.t_{k}\right) \leq \sum_{j=1}^{n} x_{i j}(t)+\frac{1}{T} \int_{0}^{T} p_{i}\left(t_{k}, x^{*}(\tau)\right) d \tau \\
& -\frac{1}{T} \int_{0}^{T} p_{i}\left(t, x^{*}(\tau)\right) d \tau \\
\leq & \frac{1}{T} \int_{0}^{T} p_{i}\left(t, x^{*}(\tau)\right) d \tau \\
& +\frac{1}{T} \int_{0}^{T} p_{i}\left(t_{k}, x^{*}(\tau)\right) d \tau \\
& -\frac{1}{T} \int_{0}^{T} p_{i}\left(t, x^{*}(\tau)\right) d \tau \\
= & \frac{1}{T} \int_{0}^{T} p_{i}\left(t_{k}, x^{*}(\tau)\right) d \tau, \quad \forall i=1, \ldots, m .
\end{aligned}
$$


Hence, $x\left(t_{k}\right) \in \mathbb{K}\left(t_{k}, x^{*}\right)$, for all $k \in \mathbb{N}$, and

$$
\begin{aligned}
& \lim _{k \rightarrow+\infty} x_{i j}\left(t_{k}\right) \\
&=\underline{x}_{i j}(t)+\min \left\{x_{i j}(t)-\underline{x}_{i j}(t),\right. \\
&\left.\bar{x}_{i j}(t)-\underline{x}_{i j}(t), x_{i j}(t)-\underline{x}_{i j}(t)\right\} \\
&=\underline{x}_{i j}(t)+x_{i j}(t)-\underline{x}_{i j}(t)=x_{i j}(t) .
\end{aligned}
$$

Then, the proof of condition (K1) is completed.

Let us prove, now, condition (K2). Let $\left\{t_{k}\right\}_{k \in \mathbb{N}}$ be a sequence such that $t_{k} \rightarrow t$, with $t \in[0, T]$, as $k \rightarrow+\infty$. Let $\left\{x\left(t_{k}\right)\right\}_{k \in \mathbb{N}}$ be a sequence, such that $x\left(t_{k}\right) \in \mathbb{K}\left(t_{k}, x^{*}\right)$, for all $k \in \mathbb{N}$, and converging to $x(t)$, as $k \rightarrow+\infty$. We have to prove that $x(t) \in \mathbb{K}\left(t, x^{*}\right)$.

Since $x\left(t_{k}\right) \in \mathbb{K}\left(t_{k}, x^{*}\right)$, for all $k \in \mathbb{N}$,

$$
\begin{gathered}
\underline{x}_{i j}\left(t_{k}\right) \leq x_{i j}\left(t_{k}\right) \leq \bar{x}_{i j}\left(t_{k}\right), \quad \forall i=1, \ldots, m, \\
\forall j=1, \ldots, n, \forall k \in \mathbb{N}, \\
\sum_{j=1}^{n} x_{i j}\left(t_{k}\right) \leq \frac{1}{T} \int_{0}^{T} p_{i}\left(t_{k}, x^{*}(\tau)\right) d \tau, \\
\forall i=1, \ldots, m, \forall k \in \mathbb{N} .
\end{gathered}
$$

Passing to the limit in (71) as $n \rightarrow+\infty$ and taking into account the continuity assumption on the functions $\underline{x}, \bar{x}$, and $p$, we have

$$
\underline{x}_{i j}(t) \leq x_{i j}(t) \leq \bar{x}_{i j}(t), \quad \forall i=1, \ldots, m, \forall j=1, \ldots, n .
$$

Now, passing to the limit for $n \rightarrow+\infty$ in the left-hand side of (72), we have

$$
\lim _{n \rightarrow+\infty} \sum_{j=1}^{n} x_{i j}\left(t_{k}\right)=\sum_{j=1}^{n} x_{i j}(t), \quad \forall i=1, \ldots, m .
$$

Then, from (74) and (59), we obtain

$$
\sum_{j=1}^{n} x_{i j}(t) \leq \frac{1}{T} \int_{0}^{T} p_{i}\left(t, x^{*}(\tau)\right) d \tau, \quad \forall j=1, \ldots, n .
$$

As a consequence,

$$
x(t) \in \mathbb{K}\left(t, x^{*}\right),
$$

and, hence, condition (K2) is achieved.

4.2. Continuity of Solutions to Weighted Quasi-Variational Inequalities. In [2, 36-39] some continuity results for variational and quasi-variational inequalities in infinite dimensional spaces have been obtained. It is worth remarking that similar results have been proved for weighted variational and quasi-variational inequalities in nonpivot Hilbert spaces (see $[4,40])$.

Now, we show a continuity result for equilibrium solutions to the dynamic elastic oligopolistic market equilibrium problem in presence of production excesses.
Theorem 7. Let $\underline{x}, \bar{x} \in C^{0}\left([0, T], \mathbb{R}_{+}^{m n}\right)$, and let $p \in$ $C^{0}\left([0, T] \times \mathbb{R}_{+}^{m n}, \mathbb{R}_{+}^{m}\right)$ be such that

$$
\begin{gathered}
\exists \phi \in C^{0}([0, T]):\|p(t, x)\| \leq \phi(t)+\|x\|^{2}, \\
\forall x \in \mathbb{R}^{m n}, \text { in }[0, T], \\
\exists \nu \in C^{0}\left([0, T], \mathbb{R}_{+}\right):\left\|p\left(t, x_{1}\right)-p\left(t, x_{2}\right)\right\| \leq \nu\left\|x_{1}-x_{2}\right\|, \\
\forall x_{1}, x_{2} \in \mathbb{R}^{m n} \text {, in }[0, T] .
\end{gathered}
$$

Moreover, let $v \in C^{1}\left([0, T] \times \mathbb{R}_{+}^{m n}, \mathbb{R}_{+}^{m}\right)$ be a vector function satisfying assumption (iii) and such that

$$
\begin{array}{r}
\exists \gamma \in C^{0}([0, T]):\left\|\nabla_{D} v(t, x)\right\| \leq \gamma(t)+\|x\|, \\
\forall x \in \mathbb{R}^{m n}, \text { in }[0, T], \\
\exists \mu>0:\left\langle-\nabla_{D} v(t, x)+\nabla_{D} v(t, y), x-y\right\rangle \geq \mu\|x-y\|^{2}, \\
\forall x, y \in \mathbb{R}^{m n}, \text { in }[0, T] .
\end{array}
$$

Then the dynamic elastic market equilibrium distribution in presence of production excesses $x^{*} \in \mathbb{K}\left(x^{*}\right)$ is continuous in $[0, T]$.

Proof. The existence of equilibrium solution is ensured by Theorem 4. Moreover, by applying Theorem 8 in [2] and taking into account Lemma 6 , we obtain the continuity of $x^{*} \in \mathbb{K}\left(x^{*}\right)$ in $[0, T]$.

\section{A Sensitivity Result}

In this section a theorem about the sensitivity of solution is presented. The following result establishes that a small change in profit function produces a small change in equilibrium distribution.

Theorem 8. Assume that the profit function changes from $v(\cdot)$ to the perturbed function $\widetilde{v}(\cdot)$ and denote by $x^{*}$ and $\tilde{x}$ the correspondent solutions of the following quasi-variational inequalities:

$$
\begin{array}{r}
\left\langle\left\langle-\nabla_{D^{v}}\left(x^{*}\right), x-x^{*}\right\rangle\right\rangle \geq 0, \quad \forall x \in \mathbb{K}\left(x^{*}\right), \\
\left\langle\left\langle-\nabla_{D} \widetilde{v}(\tilde{x}), x-\tilde{x}\right\rangle\right\rangle \geq 0, \quad \forall x \in \mathbb{K}\left(x^{*}\right) .
\end{array}
$$

Let $\nabla_{D} v(t, x)$ be a strongly monotone function of constant $\alpha$, namely, for all $x, y \in \mathbb{K}\left(x^{*}\right), \exists \alpha>0$ such that

$$
\left\langle\left\langle-\nabla_{D^{2}} v(x)+-\nabla_{D^{v}}(y), x-y\right\rangle\right\rangle \geq \alpha\|x-y\|_{L^{2}\left([0, T], \mathbb{R}^{m n}\right)}^{2} .
$$

Moreover, let $\nabla_{D} v$ be a Carathéodory function such that

$$
\begin{aligned}
\exists h \in L^{2}([0, T]): & \left\|\nabla_{D} v(t, x(t))\right\|_{m n} \\
& \leq h(t)+\|x(t)\|_{m n}, \text { a.e. in }[0, T] .
\end{aligned}
$$


Then, it follows that

$$
\left\|x^{*}-\tilde{x}\right\|_{L^{2}\left([0, T], \mathbb{R}^{m n}\right)} \leq \frac{1}{\alpha}\left\|-\nabla_{D} \widetilde{v}(\tilde{x})+\nabla_{D} v\left(x^{*}\right)\right\|_{L^{2}\left([0, T], \mathbb{R}^{m n}\right)} .
$$

Proof. Choosing $x(t)=\tilde{x}(t)$ in (79) and $x(t)=x^{*}(t)$ in $(80)$, by summing up the two new inequalities, we have

$$
\left\langle\left\langle-\nabla_{D} \widetilde{v}(\tilde{x})+\nabla_{D} v\left(x^{*}\right), x^{*}-\tilde{x}\right\rangle\right\rangle \geq 0
$$

By adding and subtracting $-\nabla_{D} v(\tilde{x})$ in (84), we have

$$
\begin{aligned}
& \left\langle\left\langle-\nabla_{D} \tilde{v}(\tilde{x})+\nabla_{D} v(\tilde{x}), x^{*}-\tilde{x}\right\rangle\right\rangle \\
& \quad \geq\left\langle\left\langle-\nabla_{D} v\left(x^{*}\right)+\nabla_{D} v(\tilde{x}), x^{*}-\tilde{x}\right\rangle\right\rangle .
\end{aligned}
$$

Moreover, by using the strong monotonicity, inequality (85), and the Cauchy-Schwartz inequality, we get

$$
\begin{aligned}
& \alpha\left\|x^{*}-\tilde{x}\right\|_{L^{2}\left([0, T], \mathbb{R}^{m n}\right)}^{2} \leq\left\langle\left\langle-\nabla_{D^{\prime}} v\left(x^{*}\right)+\nabla_{D} v(\tilde{x}), x^{*}-\tilde{x}\right\rangle\right\rangle \\
& \leq\left\langle\left\langle-\nabla_{D} \tilde{v}(\tilde{x})+\nabla_{D} v(\tilde{x}), x^{*}-\tilde{x}\right\rangle\right\rangle \\
& \leq \|-\nabla_{D} \widetilde{v}(\tilde{x})+\nabla_{D} v(\tilde{x}) \|_{L^{2}\left([0, T], \mathbb{R}^{m n}\right)} \\
& \quad \times\left\|x^{*}-\tilde{x}\right\|_{L^{2}\left([0, T], \mathbb{R}^{m n}\right)},
\end{aligned}
$$

from which we get

$$
\left\|x^{*}-\widetilde{x}\right\|_{L^{2}\left([0, T], \mathbb{R}^{m n}\right)} \leq \frac{1}{\alpha}\left\|-\nabla_{D} \widetilde{v}(\widetilde{x})+\nabla_{D} v\left(x^{*}\right)\right\|_{L^{2}\left([0, T], \mathbb{R}^{m n}\right)} .
$$

\section{A Numerical Example}

This section is devoted to provide a numerical example of the theoretical achievements presented.

Let us consider two firms and two demand markets, as in Figure 1 . Let $\underline{x}, \bar{x} \in L^{2}\left([0,1], \mathbb{R}^{4}\right)$ be the capacity constraints such that, a.e. in $[0,1]$,

$$
\underline{x}(t)=\left(\begin{array}{cc}
0 & \frac{2}{5} t \\
\frac{1}{2} t & 0
\end{array}\right), \quad \bar{x}(t)=\left(\begin{array}{cc}
10 t & 5 t \\
12 t & 10 t
\end{array}\right) .
$$

Let us denote

$$
\begin{gathered}
D=\left\{x \in L^{2}\left([0,1], \mathbb{R}^{4}\right): \underline{x}_{i j}(t) \leq x_{i j}(t) \leq \bar{x}_{i j}(t),\right. \\
\forall i=1,2, \forall j=1,2 \text {, a.e. in }[0,1]\} .
\end{gathered}
$$

Let $p \in L^{1}\left([0,1] \times D, \mathbb{R}^{2}\right)$ be the production function, such that, a.e. in $[0,1]$,

$$
p(t)=\left(\begin{array}{c}
6 t+2 x_{11}^{*}(t) \\
3 t+2 x_{11}^{*}(t)+x_{12}^{*}(t)
\end{array}\right) .
$$

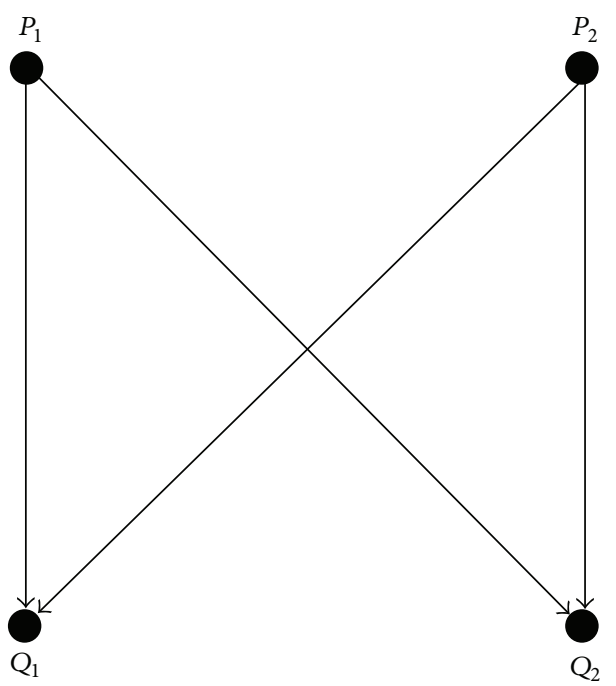

FIgURE 1: Network structure of the numerical dynamic spatial oligopoly problem.

As a consequence, the feasible set is the set value function $\mathbb{K}: D \rightarrow 2^{L^{2}\left([0,1], \mathbb{R}^{4}\right)}$ defined by

$$
\begin{gathered}
\mathbb{K}\left(x^{*}\right)=\left\{x \in L^{2}\left([0,1], \mathbb{R}^{4}\right): \underline{x}_{i j}(t) \leq x_{i j}(t) \leq \bar{x}_{i j}(t),\right. \\
\forall i=1,2, \forall j=1,2 \text {, a.e. in }[0,1], \\
\sum_{j=1}^{2} x_{i j}(t) \leq \int_{0}^{1} p_{i}\left(t, x^{*}(\tau)\right) d \tau, \\
\forall i=1,2 \text {, a.e. in }[0,1]\} .
\end{gathered}
$$

Let us consider the profit function $v \in C^{1}\left([0,1] \times D, \mathbb{R}^{2}\right)$ given by

$$
\begin{aligned}
v_{1}(t, x(t))=- & 4 x_{11}^{2}(t)-2 x_{12}^{2}(t)-x_{11}(t) x_{12}(t) \\
& +6 t x_{11}(t)+3 t x_{12}(t) \\
v_{2}(t, x(t))=- & 2 x_{21}^{2}(t)-5 x_{22}^{2}(t)-2 x_{21}(t) x_{22}(t) \\
+ & 6 t x_{21}(t)+5 t x_{22}(t) .
\end{aligned}
$$

Then, the operator $\nabla_{D} v \in L^{2}\left([0,1] \times D, \mathbb{R}^{4}\right)$ is given by

$$
\begin{aligned}
- & \nabla_{D} v(t, x(t)) \\
& =\left(\begin{array}{cc}
8 x_{11}(t)+x_{12}(t)-6 t & 4 x_{12}(t)+x_{11}(t)-3 t \\
4 x_{21}(t)+2 x_{22}(t)-6 t & 10 x_{22}(t)+2 x_{21}(t)-5 t
\end{array}\right) .
\end{aligned}
$$


The dynamic oligopolistic market equilibrium distribution in presence of the excesses is the solution to the evolutionary quasi-variational inequality:

$$
\begin{array}{r}
\int_{0}^{1} \sum_{i=1}^{2} \sum_{j=1}^{2}-\frac{\partial v_{i}\left(t, x^{*}(t)\right)}{\partial x_{i j}}\left(x_{i j}(t)-x_{i j}^{*}(t)\right) d t \geq 0, \\
\forall x \in \mathbb{K}\left(x^{*}\right) .
\end{array}
$$

Let us observe that all the hypotheses of Theorem 4 are satisfied; hence, evolutionary quasi-variational inequality (94) admits solutions.

In order to compute a solution to (94) we make use of the direct method (see [41]). We consider the following system:

$$
\begin{gathered}
8 x_{11}^{*}(t)+x_{12}^{*}(t)-6 t=0, \quad x_{11}^{*}(t)+4 x_{12}^{*}(t)-3 t=0, \\
4 x_{21}^{*}(t)+2 x_{22}^{*}(t)-6 t=0, \quad 2 x_{21}^{*}(t)+10 x_{22}^{*}(t)-5 t=0, \\
\underline{x}_{i j}(t) \leq x_{i j}^{*}(t) \leq \bar{x}_{i j}(t), \quad \forall i=1,2, \forall j=1,2, \text { a.e. in }[0,1], \\
\sum_{j=1}^{2} x_{i j}^{*}(t) \leq \int_{0}^{1} p_{i}\left(t, x^{*}(\tau)\right) d \tau, \quad \forall i=1,2, \text { a.e. in }[0,1],
\end{gathered}
$$

and we get the following solution, a.e. in $[0,1]$,

$$
x^{*}(t)=\left(\begin{array}{cc}
\frac{7}{10} t & \frac{3}{5} t \\
\frac{25}{18} t & \frac{2}{9} t
\end{array}\right) .
$$

Let us observe that the solution satisfies all the constraints; in particular, if we compute

$$
\begin{aligned}
& \int_{0}^{1} p_{1}\left(t, x^{*}(\tau)\right) d \tau=6 t+\frac{7}{10} \\
& \int_{0}^{1} p_{2}\left(t, x^{*}(\tau)\right) d \tau=3 t+1
\end{aligned}
$$

we are able to obtain the related production excesses:

$$
\epsilon(t)=\left(\begin{array}{c}
\frac{47 t+7}{10} \\
\frac{25 t+18}{18}
\end{array}\right) .
$$

\section{Conclusions}

In [1] the dynamic oligopolistic market equilibrium problem was studied by introducing production excesses, and the dynamic Cournot-Nash equilibrium was characterized as a solution to a suitable evolutionary variational inequality. In this paper, in order to have a model closer to reality, it was supposed that the production function depends on the equilibrium commodity shipment. Hence, an elastic formulation was introduced that leads to an equivalent formulation by means of a suitable evolutionary quasi-variational inequality.
By means of this mathematical formulation, results of existence and regularity of solutions were proved. Furthermore, a sensitivity analysis is provided. At last a numerical example was provided in order to clarify the theoretical results. In future work, it is possible to consider also demand excesses and elastic demand function, in order to have a more complete and realistic model.

\section{Conflict of Interests}

The authors declare that there is no conflict of interests regarding the publication of this paper.

\section{Acknowledgment}

This work has been partially supported by F.A.R.O. 2012 "Metodi matematici per la modellizzazione di fenomeni naturali."

\section{References}

[1] A. Barbagallo and P. Mauro, "Evolutionary variational formulation for oligopolistic market equilibrium problems with production excesses," Journal of Optimization Theory and Applications, vol. 155, no. 1, pp. 288-314, 2012.

[2] A. Barbagallo, "Regularity results for evolutionary nonlinear variational and quasi-variational inequalities with applications to dynamic equilibrium problems," Journal of Global Optimization, vol. 40, no. 1-3, pp. 29-39, 2008.

[3] L. Scrimali, "Quasi-variational inequalities in transportation networks," Mathematical Models \& Methods in Applied Sciences, vol. 14, no. 10, pp. 1541-1560, 2004.

[4] A. Barbagallo and S. Pia, "Weighted quasi-variational inequalities in non-pivot Hilbert spaces and applications," accepted on Journal of Optimization Theory and Applications.

[5] A. Cournot, "Researches into the mathematical principles of the theory of wealth," Competition Policy International, vol. 4, no. 1, pp. 283-305, 2008.

[6] J. F. Nash, Jr., "Equilibrium points in n-person games," Proceedings of the National Academy of Sciences of the United States of America, vol. 36, pp. 48-49, 1950.

[7] J. Nash, "Non-cooperative games," Annals of Mathematics. Second Series, vol. 54, pp. 286-295, 1951.

[8] S. Dafermos and A. Nagurney, "Oligopolistic and competitive behavior of spatially separated markets," Regional Science and Urban Economics, vol. 17, no. 2, pp. 245-254, 1987.

[9] A. Nagurney, "Algorithms for oligopolistic market equilibrium problems," Regional Science and Urban Economics, vol. 18, no. 3, pp. 425-445, 1988.

[10] A. Nagurney, P. Dupuis, and D. Zhang, "A dynamical systems approach for network oligopolies and variational inequalities," The Annals of Regional Science, vol. 28, no. 3, pp. 263-283, 1994.

[11] A. Nagurney, Network Economics: A Variational Inequality Approach, vol. 1 of Advances in Computational Economics, Kluwer Academic Publishers, Dordrecht, The Netherlands, 1993.

[12] A. Barbagallo and M.-G. Cojocaru, "Dynamic equilibrium formulation of the oligopolistic market problem," Mathematical and Computer Modelling, vol. 49, no. 5-6, pp. 966-976, 2009.

[13] M. J. Beckmann and J. P. Wallace, "Continuous lags and the stability of market equilibrium," Economica, vol. 36, pp. 58-68, 1969. 
[14] A. Barbagallo and A. Maugeri, "Memory term for dynamic oligopolistic market equilibrium problem," Aplimat_Journal of Applied Mathematics, vol. 3, no. 13, 23 pages, 2010.

[15] A. Barbagallo and R. Di Vincenzo, "Lipschitz continuity and duality for dynamic oligopolistic market equilibrium problem with memory term," Journal of Mathematical Analysis and Applications, vol. 382, no. 1, pp. 231-247, 2011.

[16] A. Barbagallo and A. Maugeri, "Duality theory for the dynamic oligopolistic market equilibrium problem," Optimization, vol. 60, no. 1-2, pp. 29-52, 2011.

[17] P. Daniele, S. Giuffrè, G. Idone, and A. Maugeri, "Infinite dimensional duality and applications," Mathematische Annalen, vol. 339, no. 1, pp. 221-239, 2007.

[18] P. Daniele and S. Giuffrè, "General infinite dimensional duality and applications to evolutionary network equilibrium problems," Optimization Letters, vol. 1, no. 3, pp. 227-243, 2007.

[19] P. Daniele, S. Giuffré, and A. Maugeri, "Remarks on general infinite dimensional duality with cone and equality constraints," Communications in Applied Analysis for Theory and Applications, vol. 13, no. 4, pp. 567-577, 2009.

[20] A. Maugeri and F. Raciti, "Remarks on infinite dimensional duality," Journal of Global Optimization, vol. 46, no. 4, pp. 581588,2010 .

[21] M. B. Donato, “The infinite dimensional Lagrange multiplier rule for convex optimization problems," Journal of Functional Analysis, vol. 261, no. 8, pp. 2083-2093, 2011.

[22] J. M. Borwein and A. S. Lewis, "Partially finite convex programming. I. Quasi relative interiors and duality theory," Mathematical Programming, vol. 57, no. 1, pp. 15-48, 1992.

[23] J. Jahn, Introduction to the Theory of Nonlinear Optimization, Springer, Berlin, Germany, 2nd edition, 1996.

[24] M. B. Donato, A. Maugeri, M. Milasi, and C. Vitanza, "Duality theory for a dynamic Walrasian pure exchange economy," Pacific Journal of Optimization, vol. 4, no. 3, pp. 537-547, 2008.

[25] M. B. Donato and M. Milasi, "Lagrangean variables in infinite dimensional spaces for a dynamic economic equilibrium problem," Nonlinear Analysis: Theory, Methods \& Applications, vol. 74, no. 15, pp. 5048-5056, 2011.

[26] A. Barbagallo and P. Mauro, "Time-dependent variational inequality for an oligopolistic market equilibrium problem with production and demand excesses," Abstract and Applied Analysis, vol. 2012, Article ID 651975, 35 pages, 2012.

[27] A. Barbagallo and P. Mauro, "On solving dynamic oligopolistic market equilibrium problems in presence of excesses," Communications in Applied and Industrial Mathematics, vol. 3, no. 1, pp. 1-20, 2012.

[28] A. Barbagallo, "Advanced results on variational inequality formulation in oligopolistic market equilirbrium problem," Filomat, vol. 5, pp. 935-947, 2012.

[29] A. Barbagallo and P. Mauro, "Inverse variational inequality approach and applications," submitted.

[30] A. Barbagallo and P. Mauro, "An inverse problem for the dynamic oligopolistic market equilibriumproblem in presence of excesses," accepted on Procedia-Social and Behavioral Sciences.

[31] O. L. Mangasarian, "Pseudo-convex functions," Journal of the Society for Industrial and Applied Mathematics, Series A Control, vol. 3, pp. 281-290, 1965.

[32] N. X. Tan, "Quasi-variational inequality in topological linear locally convex Hausdorff spaces," Mathematische Nachrichten, vol. 122, pp. 231-245, 1985.
[33] K. Kuratowski, Topology. Vol. I, Academic Press, New York, NY, USA, 1966

[34] G. Salinetti and R. J.-B. Wets, "On the convergence of sequences of convex sets in finite dimensions," SIAM Review, vol. 21, no. 1, pp. 18-33, 1979.

[35] G. Salinetti and R. J.-B. Wets, "Addendum "On the convergence of convex sets infinite dimensions"', SIAM Review, vol. 22, p. 86, 1980.

[36] A. Barbagallo, "Regularity results for time-dependent variational and quasi-variational inequalities and application to the calculation of dynamic traffic network," Mathematical Models \& Methods in Applied Sciences, vol. 17, no. 2, pp. 277-304, 2007.

[37] A. Barbagallo, "Existence and regularity of solutions to nonlinear degenerate evolutionary variational inequalities with applications to dynamic network equilibrium problems," Applied Mathematics and Computation, vol. 208, no. 1, pp. 1-13, 2009.

[38] A. Barbagallo, "On the regularity of retarded equilibrium in time-dependent traffic equilibrium problems," Nonlinear Analysis: Theory, Methods \& Applications, vol. 71, no. 12, pp. e2406e2417, 2009.

[39] A. Barbagallo and M.-G. Cojocaru, "Continuity of solutions for parametric variational inequalities in Banach space," Journal of Mathematical Analysis and Applications, vol. 351, no. 2, pp. 707720, 2009.

[40] A. Barbagallo and S. Pia, "Weighted variational inequalities in non-pivot Hilbert spaces with applications," Computational Optimization and Applications, vol. 48, no. 3, pp. 487-514, 2011.

[41] A. Maugeri, "Convex programming, variational inequalities, and applications to the traffic equilibrium problem," Applied Mathematics and Optimization, vol. 16, no. 2, pp. 169-185, 1987. 


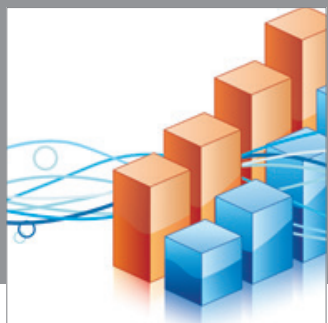

Advances in

Operations Research

mansans

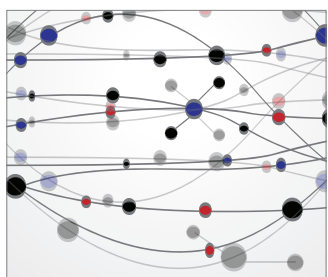

The Scientific World Journal
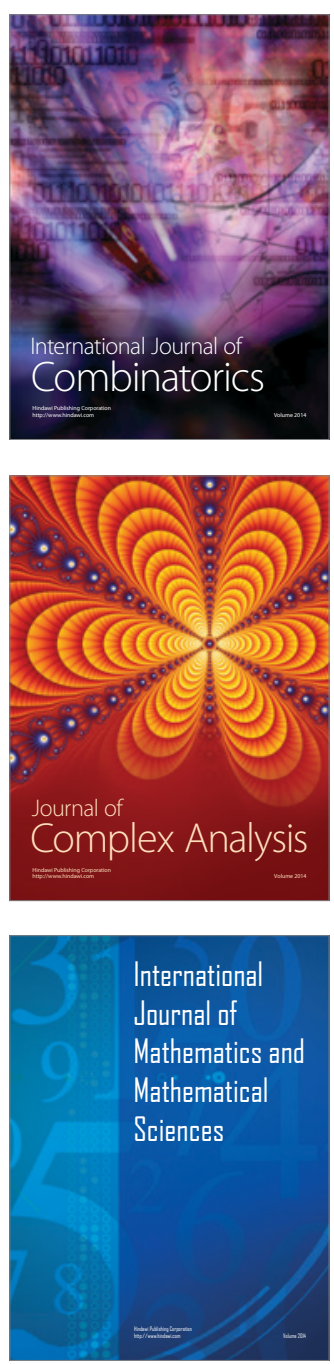
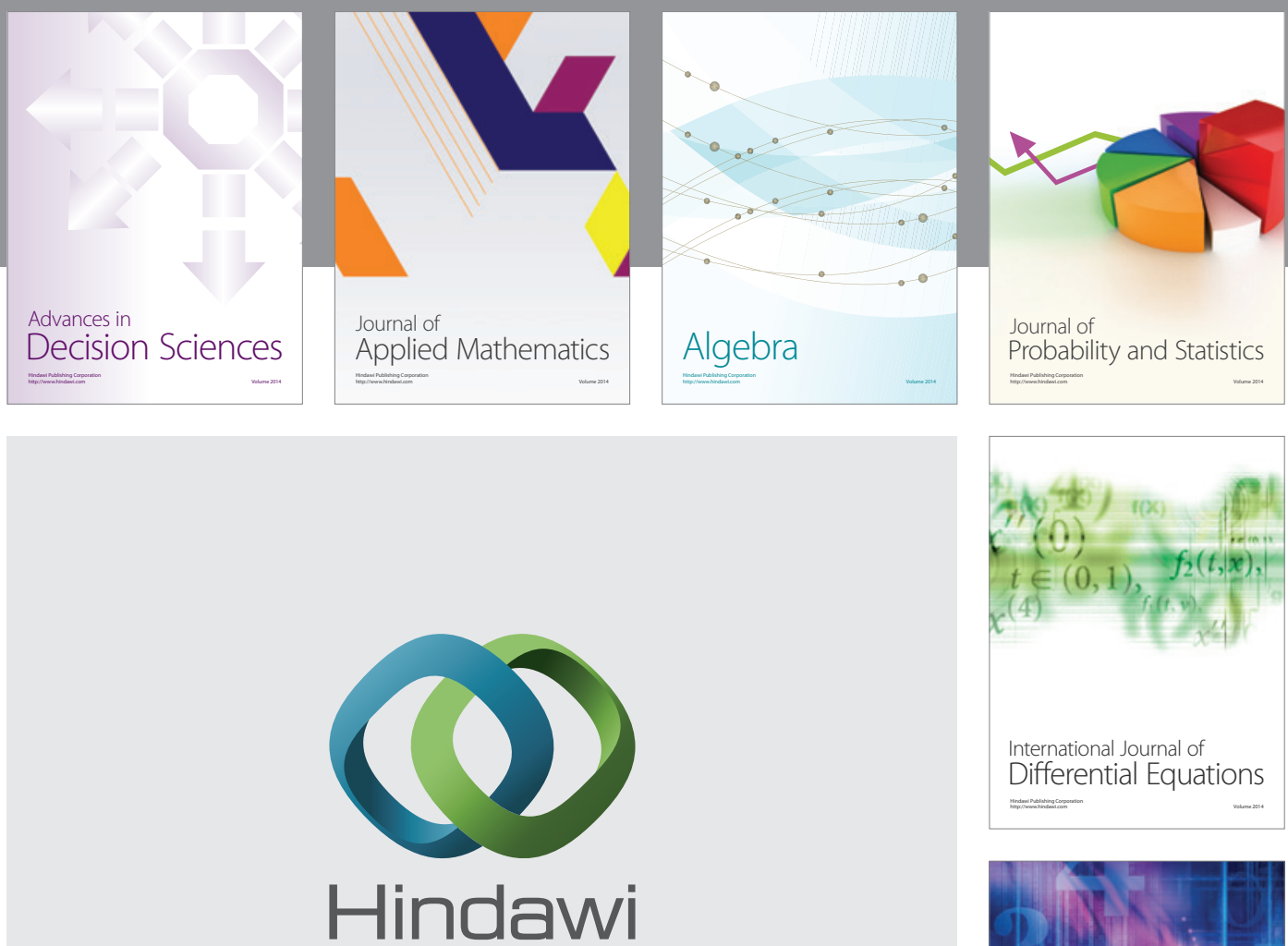

Submit your manuscripts at http://www.hindawi.com
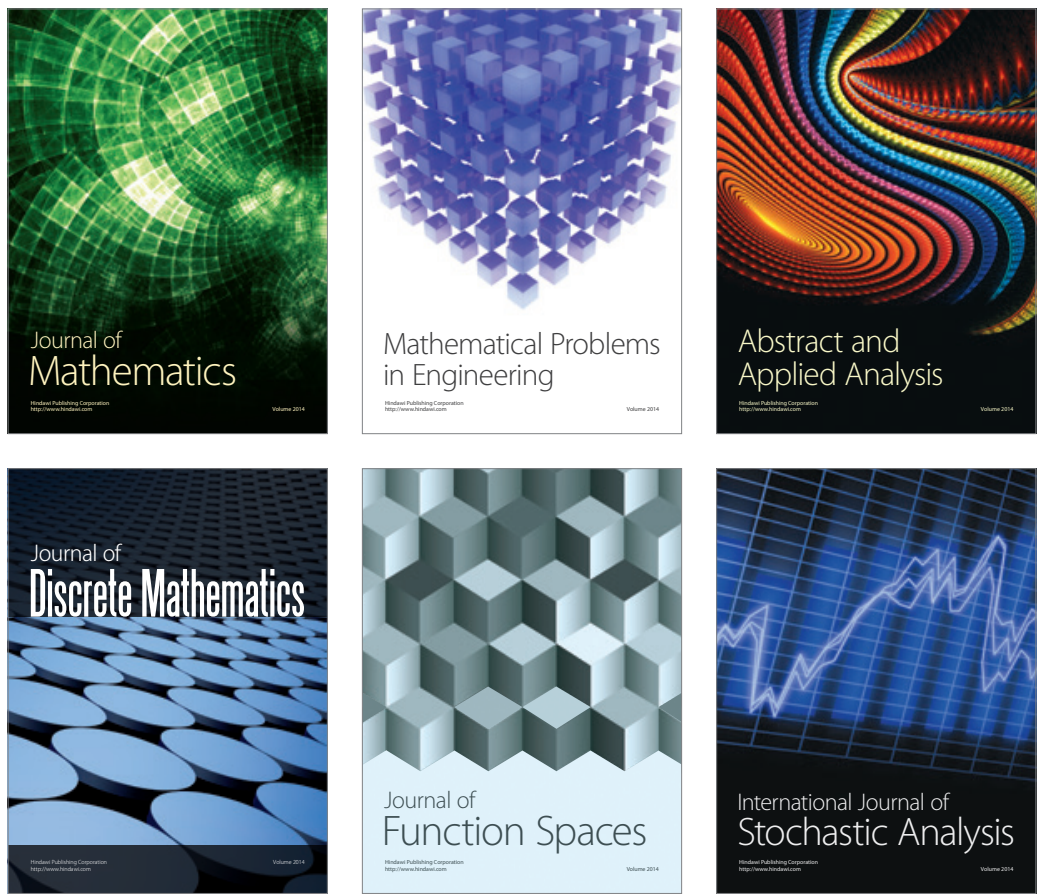

Journal of

Function Spaces

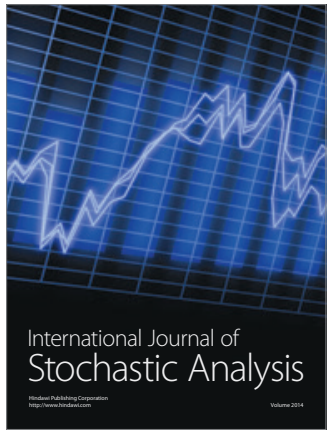

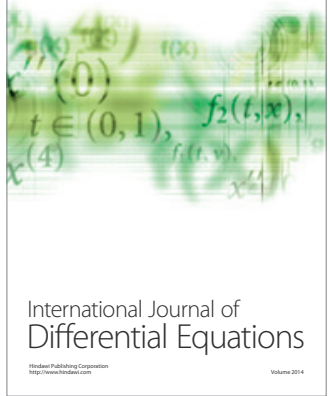
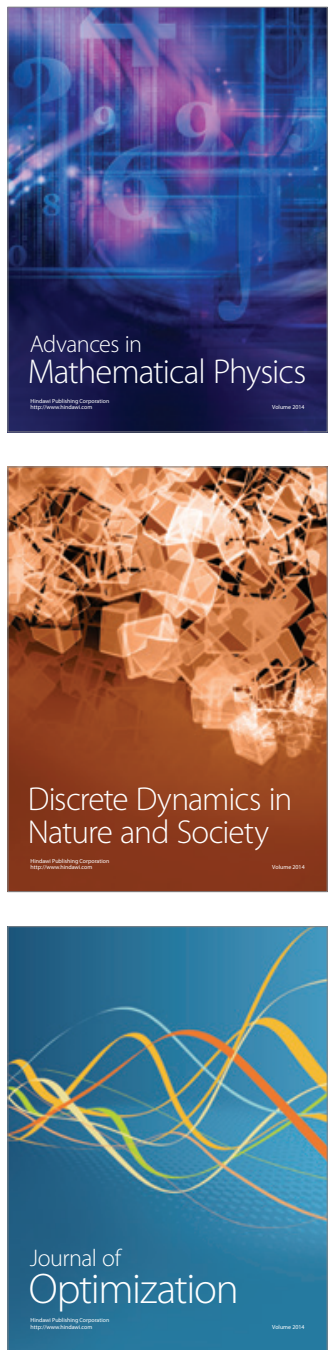